\title{
Prise en charge hospitalière: quo vadis?
}

\section{Pierre-François Cuénoud}

Dr med., ancien vice-président de la FMH et responsable du département Tarifs et économie de la santé pour les médecins hospitaliers

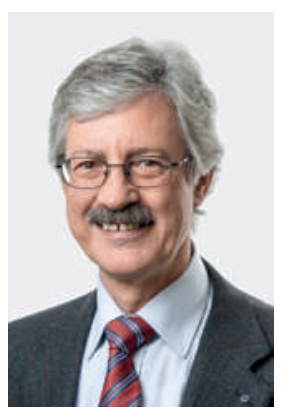

Un peu plus de quatre ans après la réforme de leur financement, les hôpitaux de notre pays sont placés devant des défis croissants, leur équilibre financier étant de plus en plus difficile à maintenir. Les causes en sont multiples, chaque intervenant se renvoyant la balle des responsabilités.

Au niveau de la structure tarifaire SwissDRG, les adaptations sont rapides d'une version à l'autre, néanmoins tributaires de la qualité des données fournies. Pour les prestations fréquentes et routinières, les poids relatifs de coûts sont maintenant réalistes. En revanche, les situations plus extrêmes sont souvent mal rétribuées.

La pression ne cesse de s'amplifier, particulièrement sur les cadres auxquels des progrès chiffrés sont constamment demandés.

Ceci est particulièrement vrai pour les cas hautement déficitaires, dont l'envergure ne peut plus être saisie par un système forfaitaire où les pertes sont compensées par certains bénéfices. Les énormes déficits rencontrés dans certaines situations doivent ainsi pouvoir être comblés de manière honnête. A cet effet, plusieurs pistes ont étés explorées par SwissDRG SA (cf. article de P. Ingenpass et B. Rohner en page 730).

Malgré de substantiels progrès depuis 2012, la version 6.0 de la structure tarifaire n'est pas encore suffisamment représentative pour éviter une nécessaire compensation dans la négociation des taux de base. Cette manière de faire n'est cependant licite que dans la phase d'introduction, dont la durée n’a pas été précisée. La marge de manœuvre avant une intervention de la Confédération est donc limitée et les modalités de la convergence de ces taux de base devront bientôt être définies. Ayant gagné son autonomie, SwissDRG doit continuer à se développer tout en gardant l'œil ouvert sur le devenir des structures tarifaires similaires à l'étranger.

Concrètement, nos hôpitaux font face à toutes sortes de difficultés. Pour pouvoir taxer certaines prestations, un nombre croissant de reconnaissances est requis dont certains critères sont particulièrement sévères et parfois ressentis comme un luxe non indispensable. Les prestations ambulatoires ne sont plus rentables et, dans le contexte général des incertitudes liées à l'avenir du TARMED, les incitations à effectuer rapidement et sans hospitalisation certaines pratiques manquent cruellement. Le soutien de l'Etat, compétent pour honorer les prestations d'intérêt général, se fait toujours plus parcimonieux.

Dans les équipes médicales, la pression ne cesse de s'amplifier, particulièrement sur les cadres responsables auxquels des progrès chiffrés sont constamment demandés. Nos collègues hospitaliers ressentent ainsi une grande lassitude, n'ayant que peu de moyens d'influencer le chiffre d'affaires de leur service sans tomber dans le piège des procédures inutiles. Face à l'augmentation constante de la bureaucratie, ils ont jusqu'à ce jour absorbé ce surplus sans prétériter le temps consacré à leurs patients, comme le montrent nos enquêtes récurrentes.

\section{Tout en sachant s'accommoder des} contingences économiques, nous devons lutter pour le maintien d'une éthique médicosoignante forte.

Alors que faire?

- Accepter de se remettre en question pour éviter toute pratique superflue, non étayée par l'évidence (qualité des indications, Health Technology Assessment);

- affiner les processus, miser sur la simplicité et le bon sens (Lean Management);

- séparer l'activité ambulatoire dans un module plus léger;

- avoir une vision stratégique à long terme, savoir sortir d'une comptabilité intransigeante répétitive pour oser investir dans l'avenir.

De manière générale, nous devons lutter pour le maintien d'une éthique médico-soignante forte: tout en sachant s'accommoder des contingences économiques, nous ne céderons rien quand il s'agit de la sécurité et des chances de guérison auxquelles chaque patient à droit. 